\title{
A NOTE ON N-CONTINUUM
}

\author{
S. GANGULY AND S. JANA
}

\begin{abstract}
In this paper a new concept allied to 'continuum' has been introduced with the name $\mathrm{N}$-continuum. Some very interesting results have been obtained which describe some interesting features of this new concept.
\end{abstract}

\section{Introduction}

In [6] S. Ganguly and T. Bandyopadhyay introduced a new type of space called 'Hcontinuum' by combining together the concepts of $\mathrm{H}$-closedness and $\theta$-connectedness ; the study was further continued in [5].

In the present paper, we utilize the concept of N-closedness [4] and $\delta$-connectedness [8] to give rise to another continuum-like concept, called N-continuum and study some of its properties.

For such study a locally nearly compact [2] space has been utilized ; in this context, concepts of $\delta$-component and $\delta$-quasicomponent have been introduced. Finally, it has been shown that the two coincide in a locally nearly compact space.

\section{Prerequisites}

Let $(X, \tau)$ be a topological space. Let $\bar{A}$ and $A^{0}$ denote the closure and interior of $A$ respectively in this space. We shall write simply $X$ to denote the topological space $(X, \tau)$, if no confusion regarding the topology arises.

\subsection{Preliminary definitions}

Definition 2.1.1([4]) A subset $A \subseteq X$ is said to be regular open (regular closed) if $A=(\bar{A})^{0}\left[\right.$ respectively $\left.A=\overline{\left(A^{0}\right)}\right]$.

Definition 2.1.2.([12]) A point $x \in X$ is said to be a $\delta$-cluster point of $A(\subseteq X)$ if $U \cap A \neq \Phi$, for every regular open neighbourhood (nbd. in short) $U$ of $x$ in $X$.

Received June 23, 2003; revised December 11, 2003.

2000 Mathematics Subject Classification. 54D20, 54D99.

Key words and phrases. Regular open, $\delta$-open, N-closedness, $\delta$-connectedness, nearly-compact, locally nearly-compact. 
The set of all $\delta$-cluster points of $A(\subseteq X)$ is called the $\delta$-closure of $A$ and we denote this by $\bar{A}^{\delta}$.

A set $A(\subseteq X)$ is said to be $\delta$-closed if $A=\bar{A}^{\delta}$.

A set $A(\subseteq X)$ is said to be $\delta$-open if $X \backslash A$ is $\delta$-closed.

Definition 2.1.3.([4]) A space $X$ is said to be semi-regular if every point of the space has a fundamental system of regularly open nbds.

Definition 2.1.4.([7]) A space $X$ is said to be almost regular if any regularly closed set $A$ and any $x \notin A$ can be strongly separated.

Definition 2.1.5. ([4]) A space $X$ is called nearly-compact if any open cover $\left\{U_{\alpha}\right.$ : $\alpha \in \Lambda\}$ of $X$ by open sets in $X$ has a finite subfamily $\left\{U_{\alpha_{i}}: i=1, \ldots, n\right\}$ such that $X=\bigcup_{i=1}^{n}\left(\overline{U_{\alpha_{i}}}\right)^{0}$.

A set $A(\subseteq X)$ is called N-closed if any open cover $\left\{U_{\alpha}: \alpha \in \Lambda\right\}$ of $A$ by open sets in $X$ has a finite subfamily $\left\{U_{\alpha_{i}}: i=1, \ldots, n\right\}$ such that $A \subseteq \bigcup_{i=1}^{n}\left(\overline{U_{\alpha_{i}}}\right)^{0}$.

Definition 2.1.6.([2]) A space $X$ is called locally-nearly compact if each point has a nbd. whose closure is N-closed.

Definition 2.1.7.([8]) A pair $(P, Q)$ of nonempty subsets of $X$ is said to be a $\delta$ separation relative to $X$ if $\bar{P}^{\delta} \cap Q=\Phi=\bar{Q}^{\delta} \cap P$.

A subset $A$ of a space $X$ is said to be $\delta$-connected relative to $X$ if there exists no $\delta$-separation $(P, Q)$ relative to $X$ such that $A=P \cup Q$.

Definition 2.1.8.([9]) A function $f: X \longrightarrow Y$ is said to be $\delta$-continuous if for any $x \in X$ and each open nbd. $V$ of $f(x)$ in $Y, \exists$ an open nbd. $U$ of $x$ in $X$ such that $f\left((\bar{U})^{0}\right) \subseteq(\bar{V})^{0}$.

\subsection{Some useful results}

Result 2.2.1.([7]) A space $X$ is almost regular iff for each $x \in X$ and each regular open set $U$ containing $x, \exists$ a regular open set $V$ such that $x \in V \subseteq \bar{V} \subseteq U$.

Result 2.2.2. Let $\left\{A_{\alpha}: \alpha \in \Lambda\right\}$ be an arbitrary family of subsets of $X$. Then

(i) $\bigcup_{\alpha \in \Lambda}\left({\overline{A_{\alpha}}}^{\delta}\right) \subseteq{\overline{\left(\bigcup_{\alpha \in \Lambda} A_{\alpha}\right)}}^{\delta}$. Equality holds if $\Lambda$ is finite.

(ii) ${\overline{\left(\bigcap_{\alpha \in \Lambda} A_{\alpha}\right)}}^{\delta} \subseteq \bigcap_{\alpha \in \Lambda}\left({\overline{A_{\alpha}}}^{\delta}\right)$

(iii) $A \subseteq B(\subseteq X) \Rightarrow \bar{A}^{\delta} \subseteq \bar{B}^{\delta}$.

Proof. Straightforward.

Note 2.2.3. It is easy to see from the above result 2.2 .2 that, the collection of all $\delta$-open sets in $X$ form a topology. We denote this topology by $\tau^{\star}$. We also note that, the collection of all regular open sets form a basis for the topology $\tau^{\star}$. Thus, each regular open set is $\delta$-open. 
Result 2.2.4.([8]) If $(P, Q)$ be a $\delta$-separation relative to $X, A \subseteq P, B \subseteq Q$ then $(A, B)$ is a $\delta$-separation relative to $X$.

Result 2.2.5. Any pair $(U, V)$ of non-empty disjoint open subsets of $X$ is a $\delta$ separation relative to $X$.

Proof. Obvious.

Result 2.2.6.([8]) If $(P, Q)$ be a $\delta$-separation relative to $X$ and $A \subseteq X$ be $\delta$-closed with $A=P \cup Q$ then, $P, Q$ are $\delta$-closed in $X$.

Result 2.2.7([10]) If $A$ be an $N$-closed set in a $T_{2}$ space $X$ then $A$ is $\delta$-closed.

Remark 2.2.8. It is clear from definition that a set $A(\subseteq X)$ is N-closed iff every regular open cover of $A$ has a finite subcover. We now give another characterisation of N-closed sets.

Result 2.2.9. A subset $A$ of $X$ is $\mathrm{N}$-closed iff every $\delta$-open cover of $A$ has a finite subcover.

Proof. If $A$ be N-closed, then, obviously, the condition holds since each $\delta$-open set contains a regular open set [by note 2.2.3].

To prove the converse, let $\left\{U_{\alpha}: \alpha \in \Lambda\right\}$ be an arbitrary open cover of $A$. Since $U_{\alpha} \subseteq\left(\overline{U_{\alpha}}\right)^{0}, \forall \alpha \in \Lambda,\left\{\left(\overline{U_{\alpha}}\right)^{0}: \alpha \in \Lambda\right\}$ is also a cover of $A$ by regular open sets in $X$. But each regular open set being $\delta$-open [by note 2.2.3] $\left\{\left(\overline{U_{\alpha}}\right)^{0}: \alpha \in \Lambda\right\}$ has a finite subcover [by given condition]. This proves that $A$ is N-closed in $X$.

Result 2.2.10. If $B$ be a $\delta$-closed subset of an $N$-closed set $A$ in a space $X$ then $B$ is also $\mathrm{N}$-closed.

Proof. Let $\left\{U_{\alpha}: \alpha \in \Lambda\right\}$ be a $\delta$-open cover of $B$. Since $B$ is $\delta$-closed $\left\{U_{\alpha}: \alpha \in\right.$ $\Lambda\} \bigcup\{X \backslash B\}$ is a $\delta$-open cover of $X$ and hence of $A$ i.e. $\left(\bigcup_{\alpha \in \Lambda} U_{\alpha}\right) \cup(X \backslash B)=X \supseteq A$.

Since $A$ is $\mathrm{N}$-closed, by result 2.2 .9 , the above cover has a finite subcover for $A$. If this finite subcover does not contain $X \backslash B$, it will be the required finite subcover of $B$. If it contains $X \backslash B$ then excluding $X \backslash B$ from this family we get the required finite subcover of $B$. Then using the result 2.2 .9 we get the result.

Result 2.2.11.([2]) (i) $A T_{2}$ space $X$ is locally-nearly compact iff for each $N$-closed set $C$ of $X$ and each regular open set $U$ such that $C \subseteq U, \exists$ an open set $V$ in $X$ such that $\bar{V}$ is $N$-closed and $C \subseteq V \subseteq \bar{V} \subseteq U$.

(ii) $A T_{2}$ space $X$ is locally-nearly compact iff for each $x \in X$ and each regular open set $U$ such that $x \in U, \exists$ an open set $V$ in $X$ such that $\bar{V}$ is $N$-closed and $x \in V \subseteq \bar{V} \subseteq U$.

Result 2.2.12. For a subset $A$ of a space $X$, the following are equivalent.

(i) $A$ is $\delta$-connected relative to $X$.

(ii) If $(P, Q)$ is a $\delta$-separation relative to $X$ and $A \subseteq P \cup Q$ then either $A \subseteq P$ or $A \subseteq Q$. 
(iii) For any $x, y \in A, \exists a \delta$-connected set $B \subseteq A$ relative to $X$ such that $x, y \in B$.

Proof. (i) $\Longleftrightarrow$ (ii) follows from lemma $2.3[8]$.

(i) $\Longrightarrow$ (iii): Taking $B=A$ the result follows.

(iii) $\Longrightarrow$ (i): If possible let, $A$ be not $\delta$-connected. Then $\exists$ a $\delta$-separation $(P, Q)$ relative to $X$ such that $A=P \cup Q$. Let $x \in P, y \in Q$. Then by (iii), $\exists$ a $\delta$-connected set $B \subseteq A$ such that $x, y \in B$. Now $(B \cap P, B \cap Q)$ forms a $\delta$-separation of $B$ relative to $X$ [by result 2.2.4] - a contradiction.

Result 2.2.13.([8]) If $A \subseteq X$ be $\delta$-connected relative to $X$ and $A \subseteq B \subseteq \bar{A}^{\delta}$ then, $B$ is $\delta$-connected relative to $X$.

Result 2.2.14.([8]) If $f: X \longrightarrow Y$ be $\delta$-continuous and $K(\subseteq X)$ is $\delta$-connected relative to $X$ then $f(K)$ is $\delta$-connected relative to $Y$.

Result 2.2.15.([9]) If $f: X \longrightarrow Y$ be $\delta$-continuous and $K(\subseteq X)$ is $N$-closed in $X$ then $f(K)$ is $N$-closed in $Y$.

Result 2.2.16. ([11]) If $A, B$ be two disjoint $N$-closed sets in a Hausdorff space $X$ then $\exists$ two disjoint regular open sets $U, V$ of $X$ such that $A \subseteq U$ and $B \subseteq V$.

\section{Example}

We know that every compact space is nearly-compact and every nearly-compact space is $\mathrm{H}$-closed. But the converse is not true in general. However, if the space be semi-regular and almost-regular then, the above three concepts become identical. So first of all we need a suitable example of a space which is neither semi-regular nor almost-regular.

Example.([1]) Let $X=\{(x, y) \in \pi: x, y \in \mathcal{Q}$ and $y \leq 0\}$, where $\pi$ is the Euclidean plane equipped with a cartesian co-ordinate system and $\mathcal{Q}$ denotes the set of all rational numbers.

Let, $X^{\prime}=\{(x, 0) \in \pi: x \in \mathcal{Q}\}$. Then $X^{\prime} \subset X$.

Let $\tau_{X^{\prime}}$ be the subspace topology on $X^{\prime}$ relative to the usual topology inherited from the plane.

Let, $\mathcal{E}$ be the collection of all open intervals lying on the $x$-axis. We fix an irrational number $\alpha>0$. For each $U \in \mathcal{E}$ we define, $U^{+}=\left\{\left(x^{\prime}, y^{\prime}\right) \in X \backslash X^{\prime}:\right.$ the line $y-y^{\prime}=$ $\alpha\left(x-x^{\prime}\right)$ intersects $\left.U\right\}$ and $U^{-}=\left\{\left(x^{\prime}, y^{\prime}\right) \in X \backslash X^{\prime}\right.$ : the line $y-y^{\prime}=-\alpha\left(x-x^{\prime}\right)$ intersects $U\}$. Also we define, $B(z ; U, V)=\{z\} \bigcup\left(U \times\{0\} \cap X^{\prime}\right) \bigcup\left(V \times\{0\} \cap X^{\prime}\right)$, where $U, V \in \mathcal{E}$ and $z \in U^{+} \cap V^{-}$. We now define, $\mathcal{B}=\left\{B(z ; U, V): U, V \in \mathcal{E}, z \in U^{+} \cap V^{-}\right\} \bigcup \tau_{X^{\prime}}$. It is easy to verify that $\mathcal{B}$ is a basis for some topology $\tau^{\prime}$ (say) on $X$ and $\left(X, \tau^{\prime}\right)$ is Hausdorff. We note that, $\left(x^{\prime}, y^{\prime}\right) \in U^{+} \Longleftrightarrow x^{\prime}-\frac{y^{\prime}}{\alpha} \in U$ and $\left(x^{\prime}, y^{\prime}\right) \in U^{-} \Longleftrightarrow x^{\prime}+\frac{y^{\prime}}{\alpha} \in U$.

Note 3.1. In the sequel we have identified $A \subseteq \mathcal{R}$ with $A \times\{0\}$, where $A$ is any subset of the real line $\mathcal{R}$; the context shall speak for itself. 
Proposition 3.2. $\overline{B(z ; U, V)}^{X}=\left(\bar{U} \cap X^{\prime}\right) \cup\left(\bar{V} \cap X^{\prime}\right) \cup\left(\bar{U}^{+} \cup \bar{U}^{-}\right) \cup\left(\bar{V}^{+} \cup \bar{V}^{-}\right)$, [where $\bar{U}$ denotes the closure of $U$ in the usual topology on the real line $\mathcal{R}$ and $\bar{B}^{X}$ denotes the closure of $B$ in $X]$.

Proof. Let us denote the R.H.S. by $A$. Then, $(x, y) \in A$ with $y=0 \Longrightarrow(x, 0) \in$ $\left(\bar{U} \cap X^{\prime}\right) \cup\left(\bar{V} \cap X^{\prime}\right)$.

Let, $W$ be any open nbd. of $(x, 0)$ in $X$.

If $(x, 0) \in \bar{U} \cap X^{\prime}$ then $W \cap U \neq \Phi \Longrightarrow W \cap B(z ; U, V) \neq \Phi$.

If $(x, 0) \in \bar{V} \cap X^{\prime}$ then $W \cap V \neq \Phi \Longrightarrow W \cap B(z ; U, V) \neq \Phi$.

Thus, $(x, 0) \in \overline{B(z ; U, V)}^{X}$. Now, let $(x, y) \in A$ with $y \neq 0$. Then $(x, y) \in\left(\bar{U}^{+} \cup\right.$ $\left.\bar{U}^{-} \cup \bar{V}^{+} \cup \bar{V}^{-}\right)$. Let, $B((x, y) ; M, N)$ be any open nbd. of $(x, y)$ in $X$. So, $(x, y) \in$ $\bar{U}^{+} \Longrightarrow x-\frac{y}{\alpha} \in \bar{U}$. Again, $x-\frac{y}{\alpha} \in M$. Since $M$ is an open interval it follows that, $M \cap U \stackrel{\alpha}{\neq} \Phi$. Consequently, $B((x, y) ; M, N) \cap B(z ; U, V) \neq \Phi$ and hence $(x, y) \in$ $\overline{B(z ; U, V)}^{X}$. Similarly, if $(x, y) \in \bar{U}^{-}$or $\bar{V}^{+}$or $\bar{V}^{-}$arguing same as above we have, $(x, y) \in \overline{B(z ; U, V)}^{X}$. Thus $A \subseteq \overline{B(z ; U, V)}^{X}-(\mathrm{i})$

Conversely, let $(x, y) \notin A$. If $y=0$ then $(x, 0) \notin\left(\bar{U} \cap X^{\prime}\right) \cup\left(\bar{V} \cap X^{\prime}\right)$.

$\Longrightarrow \exists$ open intervals $W_{1}, W_{2}$ containing $(x, 0)$ in $X$ such that $W_{1} \cap U=\Phi$ and $W_{2} \cap V=$ $\Phi \Longrightarrow\left(W_{1} \cap W_{2}\right) \cap B(z ; U, V)=\Phi$. Therefore $(x, 0) \notin \overline{B(z ; U, V)}$. If $y \neq 0$ then $(x, y) \notin\left(\bar{U}^{+} \cup \bar{U}^{-} \cup \bar{V}^{+} \cup \bar{V}^{-}\right) \Longrightarrow x-\frac{y}{\alpha} \notin \bar{U} \cup \bar{V}$ and $x+\frac{y}{\alpha} \notin \bar{U} \cup \bar{V}=\overline{U \cup V}$. So, $\exists W_{1} \in \mathcal{E}$ containing $x-\frac{y}{\alpha}$ and $W_{2} \in \mathcal{E}$ containing $x+\frac{y}{\alpha}$ such that $W_{1} \cap(U \cup V)=\Phi$ and $W_{2} \cap(U \cup V)=\Phi \Longrightarrow B\left((x, y) ; W_{1}, W_{2}\right) \cap B(z ; U, V)=\Phi . \Longrightarrow(x, y) \notin \overline{B(z ; U, V)}^{X}$. Therefore, $\overline{B(z ; U, V)} \times \subseteq A-$ (ii)

From (i) and (ii) the result follows.

Proposition 3.3. For each $U \in \mathcal{E},{\overline{U \cap X^{\prime}}}^{X}=\left(\bar{U} \cap X^{\prime}\right) \cup\left(\bar{U}^{+} \cup \bar{U}^{-}\right)$.

Proof. Let us denote the R.H.S. by $A$. Then, $(x, y) \in A$ with $y=0 \Longrightarrow(x, 0) \in \bar{U} \cap$ $X^{\prime}$. Let $W$ be any open nbd. of $(x, 0)$ in $X$. Then $W \cap U \neq \Phi \Longrightarrow W \cap\left(U \cap X^{\prime}\right) \neq \Phi \Longrightarrow$ $(x, 0) \in{\overline{U \cap X^{\prime}}}^{X}$. Now, $(x, y) \in A$ with $y \neq 0 \Longrightarrow(x, y) \in\left(\bar{U}^{+} \cup \bar{U}^{-}\right) \Longrightarrow x+\frac{y}{\alpha} \in \bar{U}$ or $x-\frac{y}{\alpha} \in \bar{U}$. Let $B\left((x, y) ; W_{1}, W_{2}\right)$ be any open nbd. of $(x, y)$ in $X$. So, $x-\frac{y}{\alpha} \in W_{1}$ and $x+\frac{y}{\alpha} \in W_{2}$.

Therefore, $W_{1} \cap U \neq \Phi$ or $W_{2} \cap U \neq \Phi \Longrightarrow W_{1} \cap\left(U \cap X^{\prime}\right) \neq \Phi$ or $W_{2} \cap\left(U \cap X^{\prime}\right) \neq \Phi$. $\Longrightarrow B\left((x, y) ; W_{1}, W_{2}\right) \cap\left(U \cap X^{\prime}\right) \neq \Phi$. Consequently, $(x, y) \in{\overline{U \cap X^{\prime}}}^{X}$.

Thus, $A \subseteq \overline{U \cap X^{\prime}} X$.

Conversely, let $(x, y) \notin A$. If $y=0$, then $(x, 0) \notin \bar{U} \cap X^{\prime}$.

$\Longrightarrow \exists$ an open nbd. $W$ of $(x, 0)$ in $X$ such that $W \cap U=\Phi$.

$\Longrightarrow W \cap\left(U \cap X^{\prime}\right)=\Phi \Longrightarrow(x, 0) \notin{\overline{U \cap X^{\prime}}}^{X}$.

If $y \neq 0$, then $(x, y) \notin\left(\bar{U}^{+} \cup \bar{U}^{-}\right) \Longrightarrow x-\frac{y}{\alpha} \notin \bar{U}$ and $x+\frac{y}{\alpha} \notin \bar{U}$.

$\Longrightarrow \exists$ open intervals $W_{1}, W_{2}$ containing $x-\frac{y}{\alpha}, x+\frac{y}{\alpha}$ respectively such that $W_{1} \cap U=\Phi$ and $W_{2} \cap U=\Phi$.

$\Longrightarrow W_{1} \cap\left(U \cap X^{\prime}\right)=\Phi$ and $W_{2} \cap\left(U \cap X^{\prime}\right)=\Phi$. 
$\Longrightarrow B\left((x, y) ; W_{1}, W_{2}\right) \cap\left(U \cap X^{\prime}\right)=\Phi$.

$\Longrightarrow(x, y) \notin \overline{U \cap X^{\prime}}$.

Therefore, ${\overline{U \cap X^{\prime}}}^{X} \subseteq A$

From (i) and (ii) the result follows.

Proposition 3.4. (i) $\left({\overline{U \cap X^{\prime}}}^{X}\right)^{0}=\left(U \cap X^{\prime}\right) \cup\left(U^{+} \cap U^{-}\right)$, for any $U \in \mathcal{E}$. (ii) $\left(\overline{B(z ; U, V)}^{X}\right)^{0}=\left(U \cap X^{\prime}\right) \cup\left(V \cap X^{\prime}\right) \cup\left(U^{+} \cap U^{-}\right) \cup\left(V^{+} \cap V^{-}\right) \cup\left(U^{+} \cap V^{-}\right) \cup\left(U^{-} \cap V^{+}\right)$, for any $U, V \in \mathcal{E}$ with $U^{+} \cap V^{-} \neq \Phi$.

Proof. (i) Since $U \cap X^{\prime}$ is open in $X$ so $U \cap X^{\prime} \subseteq\left(\overline{U \cap X^{\prime}}\right)^{X}$. Let, $(x, y) \in U^{+} \cap U^{-}$. Then $B((x, y) ; U, U) \subseteq{\overline{U \cap X^{\prime}}}^{X}=\left(\bar{U} \cap X^{\prime}\right) \cup\left(\bar{U}^{+} \cup \bar{U}^{-}\right)$. Therefore $(x, y) \in\left(\overline{U \cap X^{\prime}}{ }^{X}\right)^{0}$. Thus, $\left(U \cap X^{\prime}\right) \cup\left(U^{+} \cap U^{-}\right) \subseteq\left(\overline{U \cap X^{\prime}}\right)^{X}$. Conversely let, $(x, y) \in\left(\overline{U \cap X^{\prime}}{ }^{X}\right)^{0}$. If $y=0, \exists$ an open nbd. $W \cap X^{\prime}$ of $(x, 0)$ such that $(x, 0) \in W \cap X^{\prime} \subseteq\left(\overline{U \cap X^{\prime}}{ }^{X}\right) \Longrightarrow$ $(x, 0) \in W \cap X^{\prime} \subseteq \bar{U} \cap X^{\prime}$. If $y \neq 0, \exists$ an open nbd. $B\left((x, y) ; W_{1}, W_{2}\right)$ of $(x, y)$ such that $B\left((x, y) ; W_{1}, W_{2}\right) \subseteq{\overline{U \cap X^{\prime}}}^{X} \Longrightarrow(x, y) \in\left(\bar{U}^{+} \cup \bar{U}^{-}\right)$and $W_{1} \subseteq \bar{U}, W_{2} \subseteq \bar{U}$. But, $(x, y) \in W_{1}^{+} \cap W_{2}^{-} \Longrightarrow x-\frac{y}{\alpha} \in W_{1} \subseteq \bar{U}$ and $x+\frac{y}{\alpha} \in W_{2} \subseteq \bar{U}$. Since $W_{1}, W_{2}$ are open intervals it follows that, $x-\frac{y}{\alpha} \in U, x+\frac{y}{\alpha} \in U$ so that $(x, y) \in U^{+} \cap U^{-}$.

Thus, $\left({\overline{U \cap X^{\prime}}}^{X}\right)^{0} \subseteq\left(U \cap X^{\prime}\right) \cup\left(U^{+} \cap U^{-}\right)$. This completes the proof.

(ii) In a similar way as in (i) we have, $\left(U \cap X^{\prime}\right) \cup\left(V \cap X^{\prime}\right) \cup\left(U^{+} \cap U^{-}\right) \cup\left(V^{+} \cap V^{-}\right) \subseteq$ $\left(\overline{B(z ; U, V)}{ }^{X}\right)^{0}$. Now, $(x, y) \in U^{+} \cap V^{-} \Longrightarrow B((x, y) ; U, V) \subseteq \overline{B(z ; U, V)}{ }^{X} \Longrightarrow(x, y) \in$ $(\overline{B(z ; U, V)})^{0}$. Similarly, $(x, y) \in U^{-} \cap V^{+} \Longrightarrow B((x, y) ; U, V) \subseteq \overline{B(z ; U, V)}^{X} \Longrightarrow$ $\left.(x, y) \in(\overline{B(z ; U, V)})^{X}\right)^{0}$. Thus, $\left(U \cap X^{\prime}\right) \cup\left(V \cap X^{\prime}\right) \cup\left(U^{+} \cap U^{-}\right) \cup\left(V^{+} \cap V^{-}\right) \cup\left(U^{+} \cap\right.$ $\left.V^{-}\right) \cup\left(U^{-} \cap V^{+}\right) \subseteq(\overline{B(z ; U, V)})^{X}$. Conversely let, $\left.(x, y) \in(\overline{B(z ; U, V)})^{X}\right)^{0}$. If $y=0$, then arguing similarly as in (i) we get, $(x, 0) \in\left(U \cap X^{\prime}\right) \cup\left(V \cap X^{\prime}\right)$. If $y \neq 0, \exists$ an open nbd. $B\left((x, y) ; W_{1}, W_{2}\right)$ of $(x, y)$ such that $B\left((x, y) ; W_{1}, W_{2}\right) \subseteq \overline{B(z ; U, V)}^{X}$ $\Longrightarrow(x, y) \in\left(\bar{U}^{+} \cup \bar{U}^{-}\right) \cup\left(\bar{V}^{+} \cup \bar{V}^{-}\right)$and $W_{1} \subseteq \bar{U} \cup \bar{V}=\overline{U \cup V}, W_{2} \subseteq \overline{U \cup V}$. But, $(x, y) \in W_{1}^{+} \cap W_{2}^{-} \Longrightarrow x+\frac{y}{\alpha} \in W_{2} \subseteq \overline{U \cup V}$ and $x-\frac{y}{\alpha} \in W_{1} \subseteq \overline{U \cup V}$. Since, $W_{1}, W_{2}$ are open intervals it follows that, $x+\frac{y}{\alpha} \in U \cup V$ and $x-\frac{y}{\alpha} \in U \cup V$ so that, $(x, y) \in\left(U^{+} \cap U^{-}\right) \cup\left(V^{+} \cap V^{-}\right) \cup\left(U^{+} \cap V^{-}\right) \cup\left(U^{-} \cap V^{+}\right)$. Therefore, $(\overline{B(z ; U, V)})^{X} \subseteq$ $\left(U \cap X^{\prime}\right) \cup\left(V \cap X^{\prime}\right) \cup\left(U^{+} \cap U^{-}\right) \cup\left(V^{+} \cap V^{-}\right) \cup\left(U^{+} \cap V^{-}\right) \cup\left(U^{-} \cap V^{+}\right)$. This completes the proof.

Since $U^{+} \cap U^{-} \neq \Phi$ for any $U \in \mathcal{E}$, it follows from proposition 3.4 that, no basic open set is regular open. Also the sets in (i) and (ii) of this proposition are regular open.

Proposition 3.5. The space $\left(X, \tau^{\prime}\right)$ is not almost regular.

Proof . Let $U=\{x \in \mathcal{R}: 0<x<1\}$. Then by above discussion $\left({\overline{U \cap X^{\prime}}}^{X}\right)^{0}$ is a regular open set. We denote, $G=\left(\overline{U \cap X^{\prime}}{ }^{X}\right)^{0}$. We show that, $G$ does not contain the closure of any basic open set contained in $G$. Let, $B$ be an arbitrary basic open set such 
that $B \subseteq G$.

Case-I: $B=\left\{(x, 0) \in Q^{2}: a<x<b\right\}, B=(a, b) \cap X^{\prime}(\subset \mathcal{R})$. Then, $\bar{B}^{X}=\left([a, b] \cap X^{\prime}\right) \cup$ $\left(\bar{V}^{+} \cup \bar{V}^{-}\right)$[by proposition 3.3], where $V=(a, b)(\subset \mathcal{R})$. Since, $B \subseteq G$ it follows that $(a, b) \subset(0,1)$ i.e. $0<a<b<1$. We choose a rational $x^{\prime}<0$ and another rational $y^{\prime}$ satisfying $\alpha\left(x^{\prime}-b\right)<y^{\prime}<\alpha\left(x^{\prime}-a\right) . \Longrightarrow\left(x^{\prime}, y^{\prime}\right) \in V^{+}$and $\left(x^{\prime}, y^{\prime}\right) \notin U^{-} \Longrightarrow\left(x^{\prime} y^{\prime}\right) \in \bar{B}^{X}$ but $\left(x^{\prime}, y^{\prime}\right) \notin G$ [since $y^{\prime} \neq 0$ and by proposition 3.4]. Thus, $\bar{B}^{X} \nsubseteq G$.

Case-II: $B=B(z ; U, V)$. Since by case-I, $U^{+} \nsubseteq G$ so from proposition 3.2 it follows that, $\bar{B}^{X} \nsubseteq G$. Since any regular open set contained in $G$ must contain basic open sets, it follows from above discussion that $G$ does not contain the closure of any regular open set. Therefore by result 2.2.1, the space is not almost regular.

Proposition 3.6. The space $\left(X, \tau^{\prime}\right)$ is not semi-regular.

Proof. We took the point $(1,0) \in X$ and its open nbd. $U \cap X^{\prime}$, where $U=\{x \in \mathcal{R}$ : $0<x<2\}$. We denote $U \cap X^{\prime}=G$. Any open nbd. of $(1,0)$ contained in $G$ must be of the form $V \cap X^{\prime}$, where $V \in \mathcal{E}$ and $V \subseteq U$. But we have seen earlier that no open set of the form $V \cap X^{\prime}$ is regular open. Consequently, $G$ does not contain any regular-open nbd. of $(1,0)$. This completes the proof.

\section{N-Continuum}

In this section we introduce the concept of N-continuum and study its several properties.

Definition 4.1. Let, $X$ be a $T_{2}$-space. A $\delta$-connected (relative to $X$ ) $\mathrm{N}$-closed set in $X$ is called an $\mathrm{N}$-continuum.

Theorem 4.2. The union of two $N$-continua of a $T_{2}$-space $X$, which have a point in common, is an $N$-continuum of $X$.

Proof. Let $A, B$ be two N-continua of $X$ with $A \cap B \neq \Phi$. Let $(P, Q)$ be a $\delta$ separation relative to $X$ and $A \cup B \subseteq P \cup Q$. Since $A$ is $\delta$-connected relative to $X$ so either $A \subseteq P$ or $A \subseteq Q$ [by 2.2.12]. Now, $A \subseteq P \Longrightarrow B \subseteq P$ or $A \subseteq Q \Longrightarrow B \subseteq Q$ [since $A \cap B \neq \Phi$ and $\mathrm{B}$ is $\delta$-connected relative to $X$ ]. Thus, $A \cup B \subseteq P$ or $A \cup B \subseteq Q$. Consequently $A \cup B$ is $\delta$-connected relative to $X$ [by 2.2.12]. Also, $A \cup B$ is an N-closed set in $X$, since $A, B$ are so.

Lemma 4.3. Let $A, B$ be two $\delta$-closed sets in $X$. If the sets $A \cup B$ and $A \cap B$ are $\delta$-connected relative to $X$, then the sets $A, B$ are also $\delta$-connected relative to $X$.

Proof. If possible let, $A$ be not $\delta$-connected relative to $X$. Then $\exists$ a $\delta$-separation $(P, Q)$ of $A$ relative to $X$ such that $A=P \cup Q$. Then $A \cup B=P \cup(Q \cup B)$ and $A \cap B=(P \cap B) \cup(Q \cap B)$. If $P \cap B \neq \Phi \neq Q \cap B$ then $(P \cap B, Q \cap B)$ will form a $\delta$-separation of $A \cap B$ relative to $X$ contradicting that $A \cap B$ is $\delta$-connected relative to $X$. 
If at least one of $P \cap B, Q \cap B$ be empty, say $P \cap B=\Phi$ then, we show that $(P, Q \cup B)$ will form a $\delta$-separation of $A \cup B$ relative to $X$ contradicting that $A \cup B$ is $\delta$-connected relative to $X$.

$$
\begin{aligned}
\left(\overline{Q \cup B}^{\delta}\right) \cap P & =\left(\bar{Q}^{\delta} \cup \bar{B}^{\delta}\right) \cap P=\left(\bar{Q}^{\delta} \cup B\right) \cap P[\text { since } B \text { is } \delta \text {-closed }] \\
& =\left(\bar{Q}^{\delta} \cap P\right) \cup(B \cap P)=\Phi
\end{aligned}
$$

and

$$
\bar{P}^{\delta} \cap(Q \cup B)=\left(\bar{P}^{\delta} \cap Q\right) \cup\left(B \cap \bar{P}^{\delta}\right)
$$

Now, $P \subseteq A \Longrightarrow \bar{P}^{\delta} \subseteq \bar{A}^{\delta}=A$ [since $A$ is $\delta$-closed]

$\Longrightarrow \bar{P}^{\delta} \cap B \subseteq A=P \cup Q$.

But since $\bar{P}^{\delta} \cap Q=\Phi$ so, $\bar{P}^{\delta} \cap B \subseteq P$. Again since $B \cap P=\Phi$ it follows that $\bar{P}^{\delta} \cap B=\Phi$. Therefore from $(\star) \bar{P}^{\delta} \cap(Q \cup B)=\Phi$.

Thus, the assertion is proved and the lemma is complete.

Theorem 4.4. If $A, B$ be two $N$-closed sets in a $T_{2}$-space $X$ such that $A \cup B$ and $A \cap B$ are $N$-continua of $X$, then $A, B$ are also $N$-continua of $X$.

Proof. Since N-closed sets in a $T_{2}$-space are $\delta$-closed [by 2.2.7], the result follows from the lemma 4.3 .

Theorem 4.5. If $f: X \longrightarrow Y(X, Y$ both are Hausdorff $)$ is $\delta$-continuous and $A$ is an $N$-continuum of $X$ then $f(A)$ is an $N$-continuum of $Y$.

Proof. The theorem follows from the results 2.2.14 and 2.2.15.

Theorem 4.6. If $\left\{C_{i}\right\}_{i=1}^{\infty}$ be a decreasing sequence of $N$-continua of a locally nearly compact Hausdorff space $X$ then $\bigcap_{i=1}^{\infty} C_{i}$ is also an $N$-continuum of $X$.

Proof. Let $C=\bigcap_{i=1}^{\infty} C_{i}$. Each $C_{i}$ being N-closed of the $T_{2}$-space $X$, is $\delta$-closed [by result 2.2.7] and so $C$ is $\delta$-closed [by note 2.2.3]. Thus $C$ being a $\delta$-closed subset of an $\mathrm{N}$-closed set $C_{1}$, is $\mathrm{N}$-closed [by result 2.2.10]. We claim that $C \neq \Phi$. For, otherwise $C=\Phi \Longrightarrow X \backslash \bigcap_{i=1}^{\infty} C_{i}=X \Longrightarrow \bigcup_{i=1}^{\infty}\left(X \backslash C_{i}\right)=X \supseteq C_{1}$. Now, $\left\{\left(X \backslash C_{i}\right): i=1, \ldots\right\}$ is a $\delta$-open cover of $C_{1}$ and $C_{1}$ is N-closed. So it has a finite subcover, say, $\left\{\left(X \backslash C_{i_{n}}\right)\right.$ : $n=1, \ldots, p\}$ [by result 2.2.9]. Let $k=\max \left\{i_{1}, \ldots, i_{p}\right\}$. Then $C_{1} \subseteq \bigcup_{n=1}^{p}\left(X \backslash C_{i_{n}}\right)=$ $X \backslash \bigcap_{n=1}^{p} C_{i_{n}}=X \backslash C_{k}$ [since $\left\{C_{i}\right\}$ is a decreasing sequence] $\Longrightarrow C_{1} \cap C_{k}=\Phi-\mathrm{a}$ contradiction.

We now prove that $C$ is $\delta$-connected relative to $X$.

We assume the contrary. Then $\exists$ a $\delta$-separation $(P, Q)$ relative to $X$ such that $C=$ $P \cup Q$. Now $C$ being $\delta$-closed, so are $P, Q$ in $X$ [by result 2.2.6]. Therefore $P, Q$ must be N-closed, since $C$ is so [by result 2.2.10]. Also $P, Q$ are disjoint. Hence by result 2.2.16, $\exists$ two disjoint regular open sets $U, V$ of $X$ such that $P \subseteq U, Q \subseteq V$. Therefore, $\bigcap_{i=1}^{\infty} C_{i}=$ $C=P \cup Q \subseteq U \cup V=T$ (say). Then $T$ is $\delta$-open [by note 2.2.3]. Let $x \in C$. Then $x \in T$. 
Since $T$ is $\delta$-open, by note 2.2.3, $\exists$ a regular open set $T_{x}$ such that $x \in T_{x} \subseteq T$. Since $X$ is a locally nearly compact Hausdorff space, $\exists$ an open set $W_{x}$ such that $x \in W_{x} \subseteq \overline{W_{x}} \subseteq T_{x}$ and $\overline{W_{x}}$ is N-closed [by result 2.2.11 (ii)]. Here $W_{x}$ can be taken as a regular open (and hence $\delta$-open) set [taking $\left(\overline{W_{x}}\right)^{0}$ instead of $W_{x}$ ]. Thus $\left\{W_{x}: x \in C\right\}$ is a regular open cover of the N-closed set $C$. So it has a finite subcover $\left\{W_{x_{i}}: i=1, \ldots, n\right\}$ (say). Let $W=\bigcup_{i=1}^{n} W_{x_{i}}$. Then $C \subseteq W \subseteq \bar{W} \subseteq T$ and $\bar{W}$ is N-closed. Also $W$ is $\delta$-open [by note 2.2.3]. Therefore, $T \backslash \bigcap_{i=1}^{\infty} C_{i} \supseteq \bar{W} \backslash W \Longrightarrow \bar{W} \backslash W \subseteq \bigcup_{i=1}^{\infty}\left(T \backslash C_{i}\right)$. Now, $\bar{W} \backslash W$ is $\delta$-closed and $\bar{W} \backslash W \subseteq \bar{W}$. So $\bar{W} \backslash W$ is N-closed. Also, $T \backslash C_{i}$ is $\delta$-open $\forall i$ ( since $T$ is $\delta$-open and $C_{i}$ is $\delta$-closed $\forall i)$. So, $\exists i_{1}, \ldots, i_{p}$ such that $\bar{W} \backslash W \subseteq \bigcup_{t=1}^{p}\left(T \backslash C_{i_{t}}\right)=T \backslash \bigcap_{t=1}^{p} C_{i_{t}}=T \backslash C_{n}$, where $n=\max \left\{i_{1}, \ldots, i_{p}\right\} \Longrightarrow(\bar{W} \backslash W) \cap C_{n}=\Phi=\frac{(\star)}{\bar{W}}$

Now, $C_{n}=\left(C_{n} \backslash \bar{W}\right) \cup\left(C_{n} \cap \bar{W}\right) \subseteq\left(C_{n} \backslash W\right) \cup\left(C_{n} \cap \bar{W}\right)$. We note that, $\left(C_{n} \backslash W\right)$ and $\left(C_{n} \cap \bar{W}\right)$ both are $\delta$-closed and $\left(C_{n} \backslash W\right) \cap\left(C_{n} \cap \bar{W}\right)=C_{n} \cap(\bar{W} \backslash W)=\Phi$ [ by $(\star)]$. So $\left(C_{n} \backslash W, C_{n} \cap \bar{W}\right)$ forms a $\delta$-separation relative to $X$. Since $C_{n}$ is $\delta$-connected relative to $X$ and $C_{n} \not \subset C_{n} \backslash W$ ( for, $\left.W \supseteq C \neq \Phi\right)$ so $C_{n} \subseteq C_{n} \cap \bar{W} \subseteq \bar{W} \subseteq T=U \cup V$ [ by result 2.2.12] [Infact: $C_{n} \subseteq W$ since $(\bar{W} \backslash W) \cap C_{n}=\bar{\Phi}$ ].

Now, $U, V$ being disjoint regular open, $(U, V)$ forms a $\delta$-separation relative to $X$ [by result 2.2.5]. Since $C_{n}$ is $\delta$-connected relative to $X$, either $C_{n} \subseteq U$ or $C_{n} \subseteq V$ [by result 2.2.12] $\Longrightarrow C \subseteq U$ or $C \subseteq V \Longrightarrow$ either $C \cap Q=\Phi$ or $C \cap P=\Phi-$ a contradiction.

Thus, $C=\bigcap_{i=1}^{\infty} C_{i}$ is an N-continuum of $X$.

\section{5. $\delta$-component and $\delta$-quasicomponent}

In this article we introduce the concept of $\delta$-component and $\delta$-quasicomponent and see when these two concepts become identical.

Definition 5.1. Let $A \subseteq X$. A subset $C$ of $A$ is said to be a $\delta$-component of $A$ relative to $X$ if $C$ is $\delta$-connected relative to $X$ and is not contained properly in any other $\delta$-connected relative to $X$ subset of $A$.

Definition 5.2. A subset $C \subseteq X$ is said to be $\delta$-connected between $A$ and $B$ (where $A \cup B \subseteq C$ ) if there is no $\delta$-separation $(P, Q)$ of $C$ relative to $X$ such that $A \subseteq P, B \subseteq Q, C=P \cup Q$.

Definition 5.3. We define a relation $\rho$ on $A \subseteq X$ as follows :- $(x, y) \in \rho$ iff $A$ is $\delta$-connected between $x$ and $y$.

It is easy to verify that $\rho$ is an equivalence relation and hence induces a partition on $A$. The equivalence classes of $A$ are called $\delta$-quasicomponents of $A$. We denote the $\delta$-quasicomponents of $A$ containing $x(\in A)$ as $A[x]$.

Theorem 5.4. A set $C(\subseteq X)$ is $\delta$-connected between $A$ and $B$ (when $A \cup B \subseteq C$ ) iff $C \subseteq P \cup Q$ for any $\delta$-separation $(P, Q)$ relative to $X$ implies if $A \subseteq P$ then $B \cap P \neq \Phi$.

Proof. Follows immediately from definition. 


\section{Result 5.5.}

(i) Let $x \in A$. Then $A=A[x]$ iff $A$ is $\delta$-connected relative to $X$.

(ii) If $\Phi \neq B \subseteq A \subseteq X$ then $B[x] \subseteq A[x]$, for each $x \in B$.

(iii) Let $A \subseteq X$. Then $A[x]$ is a $\delta$-component of $A$ relative to $X$ for each $x \in A$ for which $A[x]$ is $\delta$-connected relative to $X$.

Proof. (i) Immediate from definition.

(ii) If $B$ be $\delta$-connected between $x$ and $y$ and $B \subseteq A$ then $A$ will also be $\delta$-connected between $x$ and $y$.

(iii) If possible let, $\exists$ a $\delta$-connected set $C$ relative to $X$ such that $A[x] \subseteq C \subseteq A \Longrightarrow$ $C[x] \subseteq A[x]$ [by (ii) above]. Since $C$ is $\delta$-connected relative to $X$, by (i) above, $C=C[x]$. Therefore $A[x]=C$. Consequently, $A[x]$ is a $\delta$-component of $A$ relative to $X$.

Theorem 5.6. If $A$ is a $\delta$-closed subset of $X$ then $A[x]$ is $\delta$-closed in $X$.

Proof. $\overline{A[x]} \delta \subseteq \bar{A}^{\delta}=A$ [since $A$ is $\delta$-closed] —— (*)

Let $y \in A \backslash A[x]$. Then $\exists$ a $\delta$-separation $(P, Q)$ relative to $X$ such that $x \in P, y \in Q$ and $A=P \cup Q$. Therefore $A[x] \subseteq P \Longrightarrow \overline{A[x]} \delta \subseteq \bar{P}^{\delta}$. But $\bar{P}^{\delta} \cap Q=\Phi$. So $y \notin \overline{A[x]} \delta$. Therefore using (*) we can write, $\overline{A[x]} \delta \subseteq A[x]$. This completes the proof.

Theorem 5.7. Let $X$ be a locally nearly compact $T_{2}$-space and $A$ be an $N$-closed subset of $X$. Then each $\delta$-quasicomponent of $A$ relative to $X$ is a $\delta$-component of $A$ relative to $X$.

Proof. Let $x \in A$. It now suffices to prove that $A[x]$ is a $\delta$-component of $A$ relative to $X$. For this we show that $A[x]$ is $\delta$-connected relative to $X$. Then the desired conclusion will follow from result 5.5.

Let $y \in A[x]$. We construct

$$
\mathcal{F}=\{F \subseteq A: F \text { is } \delta \text {-closed in } X, x \in F \text { and } y \in F[x]\}
$$

Since $A \in \mathcal{F}, \mathcal{F} \neq \Phi$. We define a relation ' $\geq$ ' in $\mathcal{F}$ as follows :- $F_{1} \geq F_{2}\left(F_{1}, F_{2} \in \mathcal{F}\right)$ iff $F_{1} \subseteq F_{2}$. Clearly $(\mathcal{F}, \geq)$ is a poset. Let $\mathcal{T}$ be a chain in $\mathcal{F}$ and $C=\bigcap_{F \in \mathcal{T}} F$. Then $C$ is a $\delta$-closed subset of $A$ [by note 2.2.3] and hence $C$ is N-closed [by result 2.2.10], since $A$ is N-closed. Also $x, y \in C$. We want to show $y \in C[x]$ i.e. $C$ is $\delta$-connected between $x$ and $y$.

If not, $\exists$ a $\delta$-separation $(P, Q)$ relative to $X$ such that $C=P \cup Q, x \in P, y \in Q$. Then $P, Q$ are disjoint $\delta$-closed subsets of $C$ [by result 2.2.6], since $C$ is $\delta$-closed. Hence $P, Q$ are also disjoint $\mathrm{N}$-closed sets (since $P \subseteq A, Q \subseteq A$ ) [by result 2.2.10]. So, $\exists$ two regular open sets $U, V$ in $X$ such that $P \subseteq U, Q \subseteq V, U \cap V=\Phi$ [by result 2.2.16]. Since $X$ is a locally nearly compact $T_{2}$-space and $P, Q$ are $\mathrm{N}$-closed so $\exists$ two open sets $W_{1}, W_{2}$ in $X$ such that $P \subseteq W_{1} \subseteq \overline{W_{1}} \subseteq U, Q \subseteq W_{2} \subseteq \overline{W_{2}} \subseteq V$ and $\overline{W_{1}}, \overline{W_{2}}$ are Nclosed [by result 2.2.11]. Here we can assume that $W_{1}, W_{2}$ are regular open ( and hence $\delta$-open) [taking $\left(\overline{W_{1}}\right)^{0}$ instead of $W_{1}$ ]. Therefore $\bigcap_{F \in \mathcal{T}} F=C=P \cup Q \subseteq W_{1} \cup W_{2} \subseteq$ 
$\overline{W_{1} \cup W_{2}} \subseteq U \cup V=T$ (say). Therefore $T \backslash \bigcap_{F \in \mathcal{T}} F \supseteq \overline{W_{1} \cup W_{2}} \backslash W_{1} \cup W_{2} \Longrightarrow$ $\overline{W_{1} \cup W_{2}} \backslash W_{1} \cup W_{2} \subseteq \bigcup_{F \in \mathcal{T}}(T \backslash F)$. Now, $\overline{W_{1} \cup W_{2}} \backslash W_{1} \cup W_{2}$ is a $\delta$-closed subset of $\overline{W_{1} \cup W_{2}}$ which is N-closed. So $\overline{W_{1} \cup W_{2}} \backslash W_{1} \cup W_{2}$ is N-closed. Also $T \backslash F$ is $\delta$-open, $\forall F \in \mathcal{T}$ ( since $T$ is $\delta$-open and $F$ is $\delta$-closed $\forall F$ ). So $\exists$ a finite subset $\mathcal{T}_{0}$ of $\mathcal{T}$ such that $\overline{W_{1} \cup W_{2}} \backslash W_{1} \cup W_{2} \subseteq \bigcup_{F \in \mathcal{T}_{0}}(T \backslash F)=T \backslash \bigcap_{F \in \mathcal{T}_{0}} F=T \backslash F_{0}$, for some $F_{0} \in \mathcal{T}_{0}$ ( since $\mathcal{T}_{0}$ is a finite chain $) \Longrightarrow F_{0} \cap\left(\overline{W_{1} \cup W_{2}} \backslash W_{1} \cup W_{2}\right)=\Phi-(\star)$

Now, $F_{0}=\left(F_{0} \backslash \overline{W_{1} \cup W_{2}}\right) \cup\left(F_{0} \cap \overline{W_{1}}\right) \cup\left(F_{0} \cap \overline{W_{2}}\right) \subseteq\left(F_{0} \backslash W_{1} \cup W_{2}\right) \cup\left(F_{0} \cap\right.$ $\left.\overline{W_{1}}\right) \cup\left(F_{0} \cap \overline{W_{2}}\right)$. We note that, $\left(F_{0} \backslash W_{1} \cup W_{2}\right), F_{0} \cap \overline{W_{1}}, \overline{F_{0}} \cap \overline{W_{2}}$ all are $\delta$-closed and $\left(F_{0} \cap \overline{W_{1}}\right) \cap\left(F_{0} \cap \overline{W_{2}}\right)=F_{0} \cap\left(\overline{W_{1}} \cap \overline{W_{2}}\right)=\Phi$ [since $\overline{W_{1}} \subseteq U, \overline{W_{2}} \subseteq V, U \cap V=\Phi$ ]. $\left(F_{0} \cap \overline{W_{2}}\right) \cap\left(F_{0} \backslash W_{1} \cup W_{2}\right)=F_{0} \cap\left(\overline{W_{2}} \backslash W_{1} \cup W_{2}\right)=\Phi[$ by $(\star)]$

Therefore, $\left(\left(F_{0} \backslash W_{1} \cup W_{2}\right) \cup\left(F_{0} \cap \overline{W_{1}}\right), F_{0} \cap \overline{W_{2}}\right)$ forms a $\delta$ - separation relative to $X$. Also, $x \in\left(F_{0} \backslash W_{1} \cup W_{2}\right) \cup\left(F_{0} \cap \overline{W_{1}}\right)$ and $y \in F_{0} \cap \overline{W_{2}}$ [since $x \in P \subseteq \overline{W_{1}}, y \in Q \subseteq$ $\overline{W_{2}}, x, y \in F_{0}$ ]. This contradicts that $F_{0}$ is $\delta$-connected between $x$ and $y$ [by Theorem 5.4]. Therefore, $C$ is $\delta$-connected between $x$ and $y$ i.e. $y \in C[x]$. Consequently, $C \in \mathcal{F}$. Also $C$ is an upper bound of $\mathcal{T}$. Then by Zorn's lemma $\mathcal{F}$ has a maximal element $C_{0}$ (say). Since $C_{0} \in \mathcal{F}$ so $x, y \in C_{0}$.

We now show that, $C_{0}$ is $\delta$-connected relative to $X$ and $C_{0} \subseteq A[x]$. Then by result 2.2 .12 , it follows that $A[x]$ is $\delta$-connected relative to $X$. If possible let $(M, N)$ be a $\delta$-separation relative to $X$ with $C_{0}=M \cup N$. Since $y \in C_{0}[x]$ i.e. $C_{0}$ is $\delta$-connected between $x$ and $y$ so without loss of generality we assume that $x, y \in M$. Since $M$ is $\delta$-closed in $X$ with $M \subset C_{0}$ and $C_{0}$ is a maximal element of $\mathcal{F}$, so $M$ cannot be $\delta$ connected between $x$ and $y$. Consequently, $\exists$ a $\delta$-separation $\left(M^{*}, M^{* *}\right)$ relative to $X$ such that $M=M^{*} \cup M^{* *}, x \in M^{*}, y \in M^{* *}$. Then, $C_{0}=M \cup N=M^{*} \cup\left(N \cup M^{* *}\right)$. But clearly $\left(M^{*}, M^{* *} \cup N\right)$ is a $\delta$-separation of $C_{0}$ relative to $X$ with $x \in M^{*}, y \in M^{* *} \cup N$ contradicting that $y \in C_{0}[x]$.

Thus, $C_{0}$ is $\delta$-connected relative to $X$. Therefore, $C_{0}=C_{0}[x] \subseteq A[x]$ - [by result 5.5]. This completes the proof.

\section{Acknowledgement}

Thanks to the learned Referee for his valuable comments for the improvement of the paper.

\section{References}

[1] A. V. Arkenzel'skii and V. I. Ponomarev, Fundamentals of General Topology Problems and Exercises, D.Reidel Publishing Company, Holland.

[2] D. A. Carnahan, Locally nearly-compact spaces, Boll. Un. Mat. Ital. 6(1972), 146-153.

[3] K. Kuratowski, Topology (Volume I and II), Academic Press, New York and London.

[4] M. K. Singal and Asha Mathur, On nearly-compact spaces, Boll. Un. Mat. Ital. 6(1969), 702-710.

[5] S. Ganguly and S. Jana, A note on H-continuum, [communicated]. 
[6] S. Ganguly and T. Bandyopadhyay, On H-continuum, Bull. Cal. Math. Soc. 85(1993), 311-318.

[7] M. K. Singal and S. P. Arya, On almost-regular spaces, Glasnik Mat. 4 (1969), 89-99.

[8] Takashi Noiri, A weak form of connected sets, Math. Nachr. 116(1984), 7-11.

[9] Takashi Noiri, On $\delta$-continuous functions, J. Korean Math. Soc. 16(1980), 161-166.

[10] T. Noiri, Remarks on locally nearly-compact spaces, Boll. Un. Mat. Ital. 10 (1974), 36-43.

[11] T. Noiri, N-closed sets and some separation axioms, Ann. Soc. Sci. Bruxelles 88 (1974), 195-199.

[12] N. V. Velicko, H-closed topological spaces, Amer. Math. Transl. 78(1968), 103-118.

Department of Pure Mathematics, University of Calcutta, 35, Ballygunge Circular Road, Kolkata 700019, India.

E-mail: sjpm12@yahoo.co.in 